\title{
'Measuring' Electron Delocalization in $\pi$-Conjugated Systems
}

\author{
Maurizio Bruschib, Maria Grazia Giuffreda ${ }^{a}$, and Hans Peter Lüthi
}

\begin{abstract}
For the design of donor/acceptor functionalized $\pi$-conjugated compounds, electron delocalization is a widely used concept to make structure-property predictions. In this work we present a method based on the NBO analysis, which allows selected conjugation paths in these compounds to be studied. The method maps information obtained from complex quantum calculation onto simple concepts used by general chemists.
\end{abstract}

Keywords: Cross-conjugation · D/A substitution · Electron delocalization · Natural bond orbitals · Through-conjugation

\section{Introduction}

The description of electronic charge delocalized over multiple atoms as found in aromatic or in linearly $\pi$-conjugated compounds has been a fascinating topic since the discovery of benzene by Faraday [1], and the concept of its 'partial valences' of Kekulé [2] and Thiele [3]. Later, Lewis [4], Hückel [5], Dewar [6], and others developed theoretical models, many of which are still in use despite the advanced computational methods available today. Concepts such as $\pi$-conjugation and electron delocalization are still very useful to explain and to predict chemical phenomena or molecular and electronic properties. The transformation of complex information from high level computations into simple concepts which can be used on the back of an envelope appears to be a worthwhile effort.
The interest in $\pi$-conjugated compounds is not exclusively motivated by theory. Many of these compounds show very attractive chemical properties relevant to many areas of application, including materials and biochemistry. In the recent past, $\pi$-conjugated compounds have attracted considerable attention as materials for application in molecular electronics, with the vision to replace 'silicon' by 'organic materials' [7].

One important feature of $\pi$-conjugated systems is their ability to change the electronic properties in response to external stimuli (substituents, optical excitation, etc.). The properties of the donor/acceptor functionalized polyacetylene (PA) shown in Fig. 1 can be 'tuned' by the choice of functional groups or by the solvent used. But also the length of the PA chain (the 'backbone'), the use of spacers, i.e. units such as phenyl- or ethynyl-groups inserted in the oligomer chain, are additional degrees of freedom to be exploited in the design of compounds with tailored properties.
Functionalized diethynylethenes(DEEs), tetraethynylethenes (TEEs), and their oligomers of predefined length, deep in the nanometer regime, have been synthesized in the laboratory of Diederich at ETH Zurich [8]. To avoid combinatorial numbers of compounds to be investigated it is essential for organic synthesis to apply screening methods for the identification of the high potential compounds.

It is a well known fact that many of the thoughts leading to a breakthrough in chemistry were developed on the back of an envelope using simple concepts from chemical bonding (octet rule, resonance structures) or structural chemistry (steric effects, bond length pattern). To facilitate the communication between molecular modeling and physical organic chemistry (synthesis and characterization), we have developed a method that helps translate information obtained from first principles theory (in particular electron densities) to qualitative concepts such as electron delocalization and $\pi$-conjugation.

\footnotetext{
* Correspondence: Dr. H.P. Lüthi ${ }^{\mathrm{a}}$
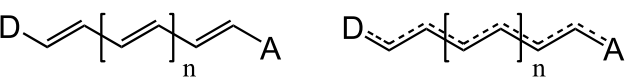<smiles>CC(C)(C=O)CCC=[V]</smiles>

Polyene

Cyanine

Zwitterion

Increasing donor /acceptor Strength

Fig. 1. Schematic representation of a functionalized $\pi$-conjugated oligomer with various degrees of bond length alternation (BLA) induced by the length of the path and the substituents. 


\section{The Method}

Various methods have been proposed for the analysis of $\pi$-conjugation in unsaturated or aromatic systems [9]. These methods range from simple bond length alternation (BLA) schemes to much more sophisticated approaches such as those based on magnetic shielding properties [10].

In the framework of molecular orbital (MO) theory, electron delocalization can be expressed as the interaction between specific occupied fragment molecular orbitals (MOs) in one part of the molecule with specific unoccupied fragment MOs in another part of the molecule. This intramolecular donor $\rightarrow$ acceptor interaction, in which electron density is transferred from one set of MOs to another, renders a basis for the quantitative description of delocalization.

We developed a method which gives a measure of $\pi$-conjugation by means of delocalization energies [11][12]. In the natural bond orbital (NBO) analysis of Weinhold [13], starting from the canonical MOs, a set of localized orbitals is obtained: the NBOs. These one center (core, lone pairs) and two center ( $\pi$ and $\sigma$ bonds) orbitals represent the 'natural Lewis structure' of the molecule. The transformation of the canonical MOs to NBOs also generates orbitals which are weakly occupied. These orbitals $\left(\sigma^{*}, \pi^{*}\right.$, Rydberg), unoccupied in the Lewis description, represent the irreducible departure from the idealized Lewis picture. Their occupation is the result of the intramolecular donor $\rightarrow$ acceptor interactions driving delocalization. Therefore, the total binding energy of a system $\left(E_{t o t}\right)$ can be partitioned into Lewis- and non-Lewis contributions $\left(E_{L}\right.$ and $\left.E_{N L}\right)$, i.e. into contributions from localized (Lewis) and delocalized (nonLewis) charge:

$E_{\text {tot }}=E_{L}+E_{N L}$

In this approach electron delocalization is determined by deletion of the weakly occupied $\pi^{*}$ NBOs and subsequent recomputation of the energy $\left(E_{\text {tot }}\right)$ in the reduced orbital space. This procedure allows a fully localized representation of the electronic structure to be obtained. Therefore we can write:

$E_{L}=E^{\prime}{ }_{t o t}$

and

$E_{N L}=E_{t o t}-E_{t o t}^{\prime}$
Under this definition, the energy associated with the deletion of the $\pi^{*}$ and $\sigma^{*}$ orbitals is a measure of delocalization. Therefore, the delocalization energy, $E_{\text {deloc }}$ ' can be obtained as:

$E_{\text {deloc }}=E_{N L}-E_{\text {strain }}-E_{\text {Rydberg }}$

where $E_{\text {strain }}$ is the energy due to steric strain and $E_{R y d b e r g}$ is the contribution to $E_{N L}$ given by Rydberg orbitals. If these two contributions are negligible, which for the applications presented here can usually be assumed, we have:

$$
E_{\text {deloc }}=E_{N L}=E_{d e l}
$$

Therefore, $E_{d e l}$ or $E_{N L}$ are equivalent to $E_{\text {deloc }}$ and may be used to quantitatively assess the delocalization energy. In addition, whenever $\sigma$ and $\pi$ orbitals are not interacting, $E_{d e l}$ can be easily broken down into $\sigma$ and $\pi$-contributions according to:

$E_{d e l}=E_{d e l}(\sigma)+E_{d e l}(\pi)$

As an example of the application of the method we have investigated the $\pi$-conjugation in butadiene. For this compound the barrier to cis-trans rotation is determined by the degree of delocalization across the single bond (the bond we rotate about). As shown in Fig. 2, the energy barrier $\left(\Delta E_{t o t}\right)$ reflects the change of the non-Lewis energy $\left(\Delta E_{N L} \approx \Delta E_{t o t}\right)$, whereas the Lewis energy remains constant between the trans form and the transition state $\left(\Delta E_{L} \approx 0\right)$. The observation that at the cis-end of the energy curves there is a deviation between the calculated rotational barrier and the nonLewis energy is a consequence of steric interaction of the hydrogen atoms in the cis form which renders Eqn. (5) invalid. For the same reason the Lewis contribution is no longer constant, but increases as the isomer approaches the cis form.

In most systems of chemical interest, however, we have several $\pi$-conjugated paths within the same molecule, and in such situations it may be important to not only determine the overall delocalization energy of the molecule but instead also quantify the delocalization energy along a specific pathway. An example is given by the geminal, trans, and cis $\pi$-conjugation pathways present in tetraethynylethene (TEE; Fig. 3) [8].

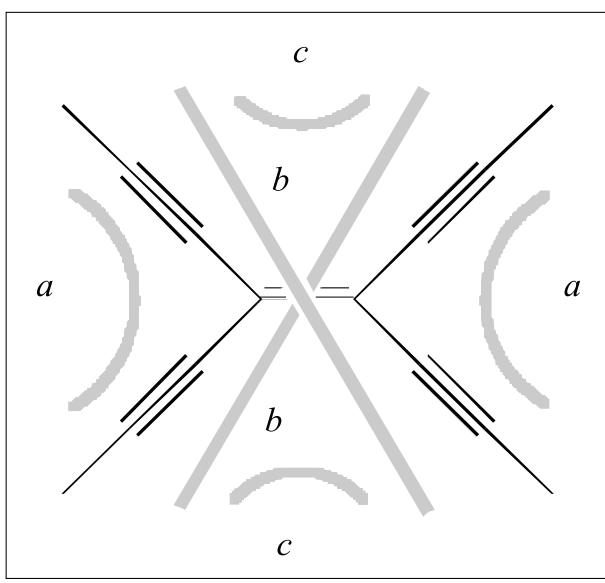

Fig. 3. Tetraethynylethene (TEE). The grey lines indicate the conjugation paths (a: geminal; b: trans; c: cis)

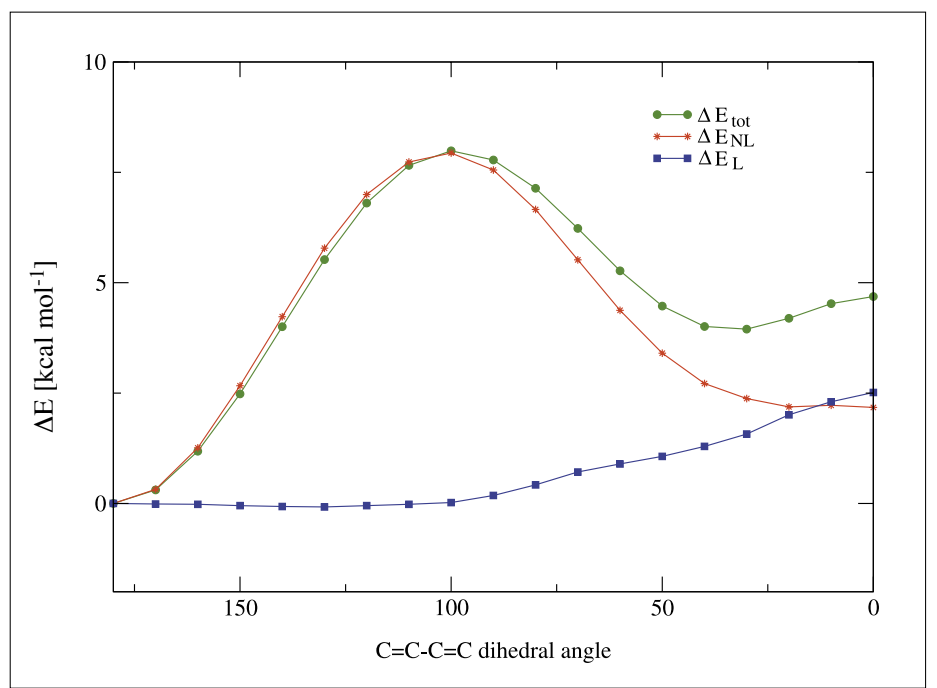

Fig. 2. The calculated $E_{t o t}, E_{L}$, and $E_{N L}$ as function of the $\mathrm{C}=\mathrm{C}-\mathrm{C}=\mathrm{C}$ dihedral angle. All energies are calculated with respect to the trans isomer (180 degrees), which is taken as the reference structure. The calculations have been performed at the B3LYP/6-31G(d,p) level of theory. 
The approach presented above can easily be extended to the 'measurement' of distinct conjugation paths. Instead of deleting all $\pi^{*}$ orbitals, only those $\pi^{*}$ orbitals conveying delocalization are being deleted. The recomputation of the energy in the reduced orbital space will show how much delocalization energy is associated with that path. In addition, the same protocol can be extended to the investigation of the in-plane $\sigma$-hyperconjugation as well.

A second useful tool for the analysis of delocalization is given by the second-order orbital interaction energies (SOIEs) which allow for a better insight into the origin of the relevant contributions to the delocalization energy. The SOIE for a pair of filled Lewis-type NBOs (donor orbitals) and weakly occupied non-Lewis-type NBOs (acceptor orbitals), labeled $i$ and $j$, is given by the formula

$$
E(2)=E_{S O I E_{i j}}=\Delta E_{i j}=q_{i} \frac{F(i, j)^{2}}{\varepsilon_{i}-\varepsilon_{j}}
$$

where $q_{i}$ is the donor orbital occupancy, $\varepsilon_{i}$ and $\varepsilon_{j}$ are the orbital energies, and $\mathrm{F}(i, j)$ is the corresponding element of the Fock matrix in the NBO basis. As long as higher order contributions can be neglected, the sum of $E_{S O I E}$ along a given pathway should correspond to $E_{N L}$

$$
\sum_{i j} E_{S O I E_{i j}}=E_{N L}
$$

A detailed description of the method is given in [12].

\section{Applications}

\subsection{Focus}

One of the most important questions in the search for high performance materials based on donor/acceptor functionalized $\pi$ conjugated systems (as shown in Fig. 1) is how a given carbon backbone is able to promote the impact of the functional groups. Or, in other words, what is the efficiency with which a given conjugation path couples the functional groups.

The most straightforward experimental evidence for a highly efficient conjugation path is the change in bond length alternation (BLA). If donor/acceptor substitution invokes a major change in BLA, we can assume that the functional groups induce a large promotion of $\pi$-delocalization in the backbone and the resulting compound might show interesting photochemical or non-linear optical properties. Optimization of the conjugation path (type and length) and substitution pattern (donor, acceptor) are the tasks to be addressed.

\subsection{Through (cis/trans) versus Cross (geminal) Conjugation}

The general view is that cross-conjugation is less efficient in promoting $\pi$-conjugation than through-conjugation. This is supported by experimental as well as by theoretical studies [11][14][15]. Indeed, the application of our method to the study of electron delocalization in through and cross conjugated diethynylethenes (see Fig. 4) shows that the delocalization energy $\left(E_{d e l}\right)$ of the trans-diethynylethene is about $5 \mathrm{kcal}$ $\mathrm{mol}^{-1}$ larger than that of the geminal isomer [11]. This value is almost exactly equal to the total energy difference $\left(\Delta E_{t o t}\right)$ between the two isomers, indicating that this energy difference between the two isomers is due to the non-Lewis contributions.

Partitioning of delocalization energies into $\sigma$ and $\pi$ contributions shows that the stability difference between cross and through conjugation is controlled by vertical $\pi$-conjugation, and that $\sigma$-hyperconjugation plays a very minor role only. However, decomposition of $E_{d e l}(\sigma)$ into different orbital contributions shows that the geminal isomer would be favored by as much as $15 \mathrm{kcal} \mathrm{mol}^{-1}$, if it was not for a hyperconjugation effect involving the $\mathrm{C}-\mathrm{H}$ $\sigma^{*}$ orbitals of the trans isomer which almost perfectly compensates the in-plane $\mathrm{C}-\mathrm{C}$ conjugation advantage of the geminal isomer. The importance of $\sigma$-conjugation is illustrated by the fact that the largest structural differences between the DEE isomers are not in the $\pi$-bond lengths but rather in the $\sigma$-bond lengths: in the trans isomer the $=\mathrm{C}-\mathrm{C} \sigma$-bonds are consistently longer than

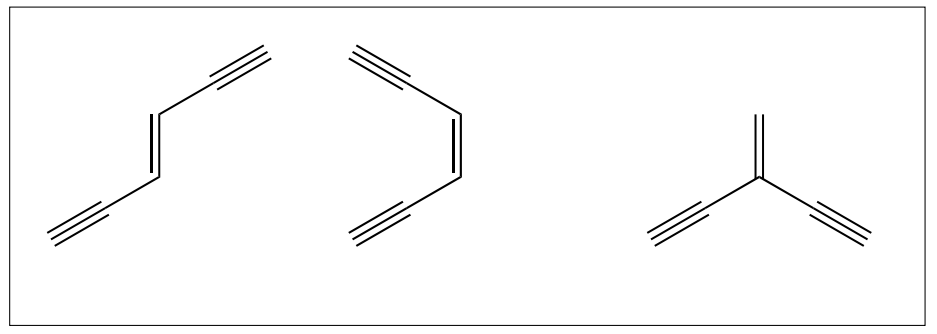

Fig. 4. Through- (trans and cis) and cross- (geminal) conjugated diethynylethenes

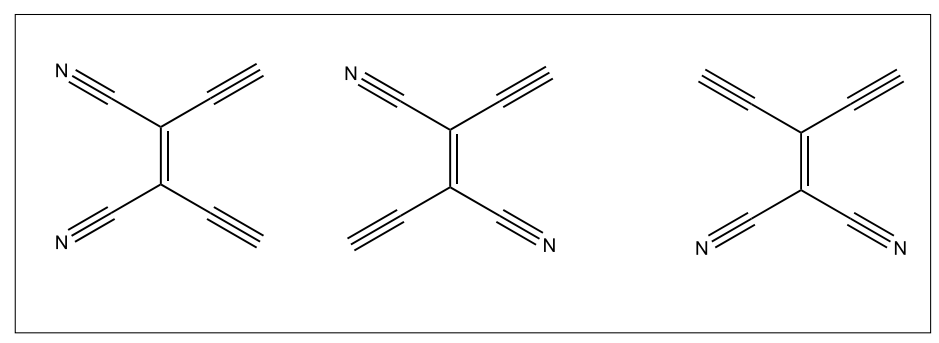

Fig. 5. The cross- and through-conjugated isomers of di-cyanoethynylethenes (2CN2Es) in the geminal isomer. This indicates that $\sigma$ conjugation cannot be neglected, and that it can be the source of discrimination between geminal and trans conjugation.

Substitution can change the situation quite drastically: replacing the two hydrogens in DEEs with cyano groups $(\mathrm{CN})$ to form di-cyanoethnynylethenes (2CN2Es, Fig. 5), leads to a geminal and a trans isomer which are extremely close in energy.

Inspection of the distinct conjugation paths in constitutional isomers of 2CN2Es (Fig. 6) reveals that the most stable path is indeed the geminal $\mathrm{CN}-\mathrm{C}-\mathrm{CN}$ (or XX) path in the cross-conjugated system, explaining the change in stability trends upon cyano substitution (for more details see [16]).

In general, cyanoethynylethenes, which due to their exceptional properties have caught considerable attention in organic material synthesis [17], provide an example where geminal $\pi$-conjugation is stronger than through conjugation.

\subsection{The Effect of Donor and Acceptor Substitution}

Application of the analysis to substituted TEEs shows that the TEE backbone responds more favorably to donor than to acceptor substitution. This observation is supported by the molecular structures: for donor-substitution a significantly larger BLA is observed. In Fig. 7 we present the delocalization energy calculated for TEE$n \mathrm{NO}_{2}$ and TEE- $n \mathrm{OH}$ with $n=1-4$, that is for TEE substituted with an acceptor and a donor, respectively.

These data show first that the delocalization energies in the TEEs substituted with donors are much larger than those in the acceptor-substituted TEEs for both through 

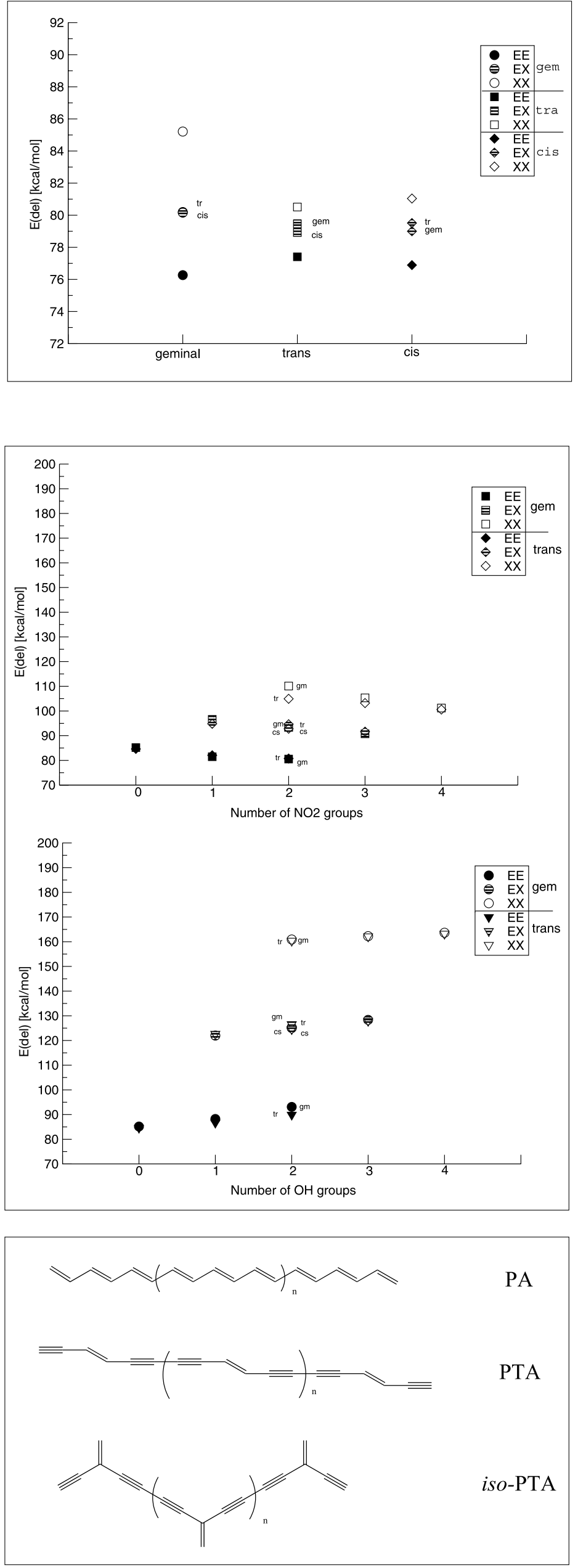

Fig. 6. Delocalization energies of distinct conjugation paths for the three constitutional isomers of the dicyanoethynylethenes (2CN2Es).

Fig. 7. The geminal and trans delocalization energies for TEE- $n \mathrm{X}$, where $\mathrm{X}$ $=\mathrm{NO}_{2}$ and $\mathrm{OH}$ and $\mathrm{n}$ $=0-4$. In the legend, EE defines paths of two ethylenic units, EX paths of one ethylenic unit and one $-\mathrm{C} \equiv \mathrm{C}-\mathrm{X}$ unit, and $X X$ paths with two $-\mathrm{C} \equiv \mathrm{C}-\mathrm{X}$ units.

Fig. 8. PTA, iso-PTA, and PA oligomers. and cross conjugated pathways. This is due to much stronger interactions of the donor lone pairs with the backbone. Second, the delocalization energies not only depend on the nature of the substituent (donor versus acceptor), but also strongly on the degree of substitution in the conjugation path. The impact of the neighboring path is still visible, but much less pronounced. In other words, the delocalization energies significantly increase with the number of substituents in a conjugation path, but show a very small variation when increasing the number of substituents in the neighbor path.

In contrast to TEEs (Fig. 6), the cynoethynylethenes are generally characterized by very small differences between through and cross conjugation (Fig. 7).

\subsection{Extended Systems: The Impact of Length and Type of Backbone}

Trans and geminal DEEs (see Fig. 4) are the building blocks of the linearly conjugated polytriacetylene (PTA) and crossconjugated iso-PTA polymers, respectively (see Fig. 8). Oligomers of PTA and isoPTA of selected chain length can be easily functionalized with donor and/or acceptor substituents to give compounds of tailored properties [8][18]. In this respect, it is of basic importance to understand how $\pi$-conjugation is promoted by backbones of different length and type. For the PTA oligomers, a response to chain length is observed, until the system finally saturates at a length of 20-22 unsaturated bonds. In the case of the iso-PTA oligomers, saturation of BLA is reached for the longest linearly conjugated segment, showing that $\pi$-conjugation is switched off at the cross-conjugated carbon atom [15][19].

The analysis shows that the vertical $\pi$ delocalization energy per unsaturated bond (Eл; see Fig. 9b) of PTA is slightly larger than that of PA. However, E $\pi$ of PA grows faster with increasing chain length. This indicates that the PA backbone promotes $\pi$-conjugation more efficiently than the PTA one. Even though they substantially contribute to the delocalization energy, the ethynyl groups appear to propagate $\pi$-conjugation less efficiently [18]. In these systems, in-plane conjugation does not play a prominent role.

The efficiency of promotion of $\pi$-conjugation in different backbones (PTA, iso-PTA, and PA) can be investigated by considering the response of the backbone to chain elongation $\left(\Delta E \pi_{\text {SOIE }}\right)$. Partitioning of the oligomer into a central backbone and into two terminal units, we can define $\Delta E \pi_{\text {SOIE }}$ as:

$$
\Delta E \pi_{\text {SOIE }}=\sum E \pi_{\text {SOIE }}^{\text {mol }}-\sum E \pi_{\text {SOIE }}^{\text {back }}
$$


where $\sum E \pi_{\text {SOIE }}^{\text {mol }}$ is the sum of the $E_{\text {SOIE }}$ energies between vertical $\pi^{*}$ orbitals of the central backbone with the two terminal units attached, and $\sum E \pi_{\text {SOIE }}^{\text {back }}$ is the sum of the $E_{S O I E}$ energies between vertical $\pi^{*}$ orbitals of the central backbone without terminal units. Therefore, $\Delta E \pi_{S O I E}$ measures the increase of $\pi$ delocalization energy of a backbone in response to the extension of the conjugation pathway.

The evolution of $\Delta E \pi_{S O I E}$ as the chain length increases clearly indicates that the incorporation of triple bonds in the PA chain to give PTA significantly reduces the efficiency of $\pi$-conjugation (see Fig. 9a). These results are in agreement with the evolution of calculated properties such as BLA and $\lambda_{\text {max }}$ and lead to the conclusion that the efficiency in promoting $\pi$-conjugation for a backbone is not related to the total amount of $\pi$-delocalization present, but to the response of the backbone to the extension of the conjugation pattern. Regarding the iso-PTA backbone, Fig. 9a shows that $\Delta E \pi_{\text {SOIE }}$ remains constant confirming that $\pi$-conjugation is switched off at the cross-conjugated carbon atom.

\section{Conclusions}

Even though just a concept, $\pi$-conjugation is still a useful tool to make structure and property predictions on the back of an envelope. This work also shows that the method of analysis presented here gives additional information on how $\pi$-conjugation extends over a carbon backbone and how efficiently it couples functional groups. In that matter, we showed that geminal conjugation is not always a loosing proposition, and that proper function- alization may make a geminal conjugated path more efficient than its through-conjugated counterparts (cis and trans).

The method presented maps relatively complex information (first-order density matrix generated by the appropriate quantum chemical method) onto quite simple concepts (local contributions to delocalization energy), in the spirit of the word saying that after every great quantum computation there is a chemical explanation.

\section{Acknowledgements}

The authors wish to acknowledge the support of Swiss National Science Foundation (through grant 200020-100346) and the Swiss National Supercomputing Center (CSCS). This work has strongly benefited from discussions with Prof. F. Weinhold, Prof. F. Diederich and Dr. D. Bakowies.

Received: June 10, 2005

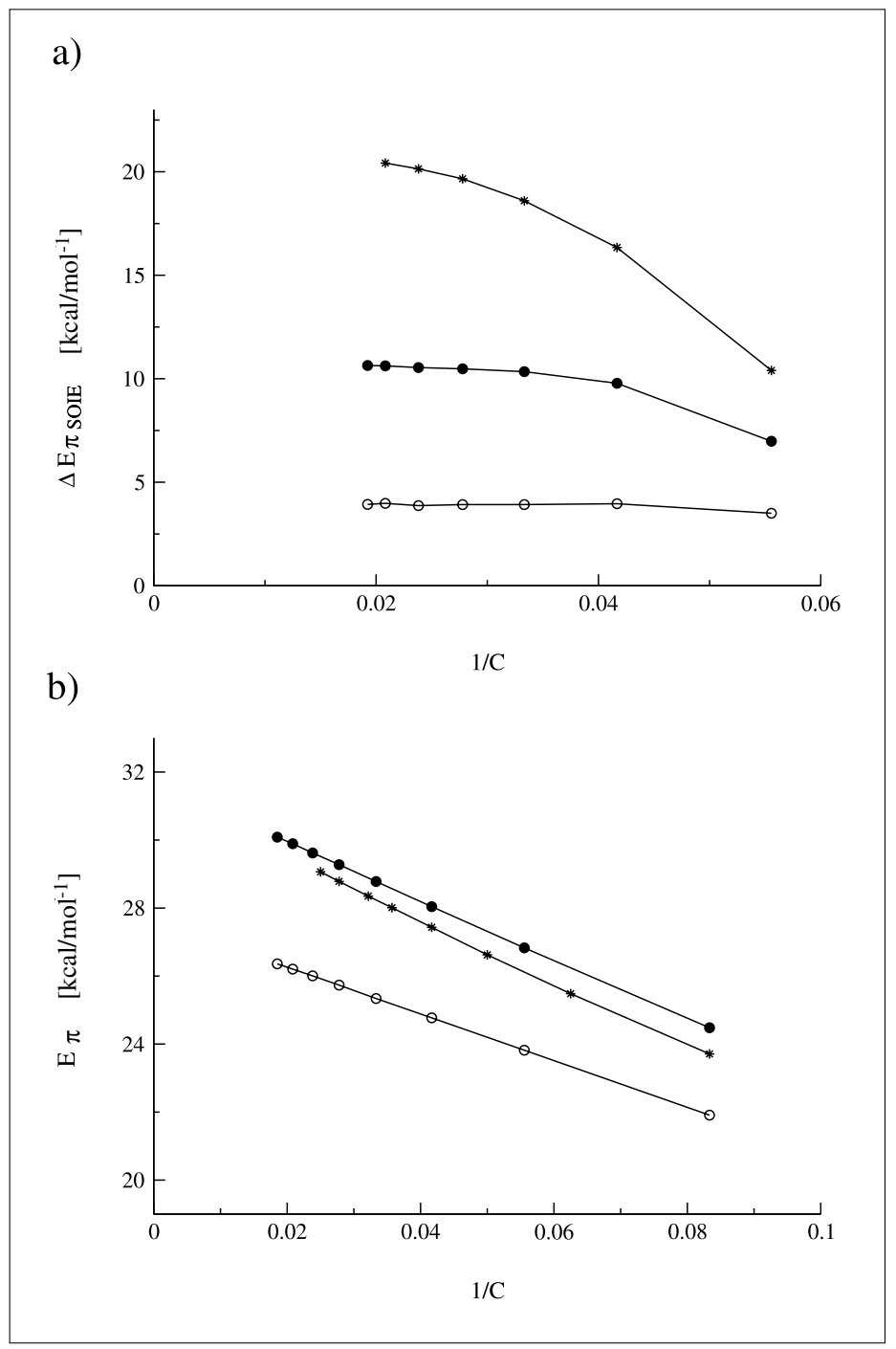

[1] M. Faraday, Phil. Trans. R. Soc. London 1825, 440 .

[2] A. Kekulé, Bull. Soc. Chim. Paris 1865, 3, 98; Bull. Acad. R. Belg. 1865, 19, 551.

[3] J. Thiele, Liebigs Ann. Chem. 1899, 306, 87.

[4] a) L. Pauling, J. Am. Chem. Soc. 1931, 53, 1367; b) J.C. Slater, Phys. Rev. 1931, 37 , 481 ; c) C.A. Coulson, 'Valence' $2^{\text {nd }} E d$. Oxford University Press, London, 1952.

[5] E. Hückel, Z. Phys. 1930, 60, 423.

[6] M.J.S. Dewar, 'The Molecular Orbital Theory of Organic Chemistry', McGrawHill, New York, 1969.

[7] a) 'Modern Acetylene Chemistry', Eds. P.J. Stang, F. Diederich, Wiley-VCH, Weinheim, 1995; b) 'Carbon Rich Compounds II, Vol. 201', Ed. A. de Meijere, Springer, Berlin, 1999; c) D.L. Pearson, J.S. Schumm, J.M. Tour, Macromolecules 1994, 27, 2348; d) P. Seta, E. Bienvenue, Mol. Electron Devices 1994, 3, 59; e) J.S. Schumm, D.L. Pearson, J.M. Tour, Angew. Chem. 1994, 106, 1445; Angew. Chem. Int Ed. Engl. 1994, 33, 1360; f) J.M. Tour, Chem. Rev. 1996, 96, 537; g) T. Bartik, B. Bartik, M. Brady, R. Dembinski, J.A. Gladsyz, Angew. Chem. 1996, 108, 467; Angew. Chem. Int. Ed. 1996, 35, 414; h) 'Handbook of Conducting Polymers, Vols. 1, 2', Ed. T.A. Skotheim, Dekker, New York, 1986; i) 'Electronic Properties of Conjugated Polymers III: Basic Models and Applications', Eds. H. Kuzmani, M. Mehring, S. Roth, Springer, Berlin, 1989; j) 'Conjugated Polymers and Related Materials. The Interconnection of Chemical and Electronic Structure', Eds. W.R. Salaneck, I. Lundström B. Ränby, Oxford University Press, Oxford, 1993; k) N.C. Greenham, S.C. Moratti, D.D.C. Bradley, R.H. Friend, A.B. Holmes, Nature 1993, 365,628 ; 1) M. Liphardt, A. Goonesekera, B.E. Jones, S. Ducharme, J.M. Takacs,

Fig. 9. a) Evolution of $\Delta E \pi_{S O / E}$, as defined by Eqn. (9), as function of reciprocal number of (backbone) carbon atoms for the PTA (•), iso-PTA (o), and PA (*) oligomers computed at the B3LYP/6-31G(d,p) level of theory. b) $E \pi$ values as function of the reciprocal number of (backbone) carbon atoms for the PTA $(\bullet)$, iso-PTA (o), and PA $\left(^{*}\right)$ oligomers computed at the B3LYP/6$31 \mathrm{G}(\mathrm{d}, \mathrm{p})$ level of theory. 
L. Zhang, Science 1994, 263, 367; m) M. Brøndsted Nielsen, F. Diederich, Chem. Rec. 2002, 2, 189.

[8] a) M. Schreiber, J. Anthony, F. Diederich, M.E. Spahr, R. Nesper, M. Hubrich, F. Bommeli, L. Degiorgi, P. Wachter, P. Kaatz, C. Bosshard, P. Günter, M. Colussi, U.W. Suter, C. Boudon, J.-P. Gisselbrecht, M. Gross, Adv. Mater. 1994, 6, 786; b) J. Anthony, A.M. Boldi, Y. Rubin, M. Hobi, V. Gramlich, C.B. Knobler, P. Seiler, F. Diederich, Helv. Chim. Acta 1995, 78, 13; c) M. Schreiber, R.R. Tykwinski, F. Diederich, R. Spreiter, U. Gubler, C. Bosshard, I. Poberaj, P. Günter, C. Boudon, J.-P. Gisselbrecht, M. Gross, U. Jonas, H. Ringsdorf, Adv. Mater. 1997, 9, 339.

[9] Y. Mo, J. Chem. Phys. 2003, 119, 1300 and references therein.

[10] C. Corminbœuf, T. Heine, J. Weber, PhysChemChemPhys 2003, 5, 246.

[11] M. Bruschi, M.G. Giuffreda, H.P. Lüthi, Chem. Eur. J. 2002, 8, 4216.

[12] M.G. Giuffreda, M. Bruschi, H.P. Lüthi, Chem. Eur. J. 2004, 10, 5671.

[13] a) T.K. Brunck, F. Weinhold, J. Am. Chem. Soc. 1978, 101, 1700; b) J.P. Foster, F. Weinhold, J. Am. Chem. Soc. 1980, 102, 7211; c) A.E. Reed, R.B. Weinstock, F. Weinhold, J. Chem. Phys. 1985, 83, 735; d) A.E. Reed, F. Weinhold, J. Chem. Phys. 1985, 83, 1736; e) A.E. Reed, L.A. Cur- tiss, F. Weinhold, Chem. Rev. 1988, 88 , 899; (f) F. Weinhold, J.E. Carpenter, 'The Structure of Small Molecules and Ions', Plenum 227, 1988.

[14] a) R.R. Tykwinski, Y. Zhao, Syn. Lett. 2002, 12, 1939; b) Y. Zhao, R. McDonald, R.R. Tykwinski, Chem. Commun. 2000, 1, 77; c) Y. Zhao, K. Campbell, R.R. Tykwinski, J. Org. Chem. 2002, 67, 2805; d) M. Klokkenburg, M. Lutz, A.L. Spek, J.H van der Maas, C.A. van Walree, Chem. Eur. J. 2003, 9, 35444.

[15] M. Bruschi, M.G. Giuffreda, H.P. Lüthi, ChemPhysChem 2005, 6, 511.

[16] M.G. Giuffreda, N.N.P. Moonen, H.P. Lüthi, F. Diederich, to be submitted to Chem.-Eur. J. 2005.

[17] a) N.N.P. Moonen, C. Boudon, J.P. Gisselbrecht, P. Seller, M. Gross, F. Diederich, Angew. Chem. Int. Ed. 2002, 41, 3044; b) N.N.P. Moonen, R. Gist, C. Boudon, J.P. Gisselbrecht, P. Seller, T. Kawai, A. Kishioka, M. Gross, M. Irie, F. Diederich, Org. Biomol. Chem. 2003, 1, 2032; c) N.N.P. Moonen, F. Diederich, Org. Biomol. Chem. 2004, 2, 2263.

[18] a) T. Ito, H. Shirakawa, S. Ikeda, Polymer J. 1971, 2, 231; b) T. Ito, H. Shirakawa, S. Ikeda, Polym. Chem. Ed. 1974, 12, 11.

[19] M. Bruschi, M.G. Giuffreda, H. P. Lüthi, to be submitted to ChemPhysChem 2005 . 